\title{
An In Vivo Bioassay for Estimating Fungicide Residues on Peach Fruit
}

\author{
N. Lalancette, J. Gager, and K. A. McFarland, Rutgers University, Department of Plant Biology and Pathology, Rutgers Agricultural \\ Research and Extension Center, Bridgeton, NJ 08302-5919
}

\begin{abstract}
Lalancette, N., Gager, J., and McFarland, K. A. 2015. An in vivo bioassay for estimating fungicide residues on peach fruit. Plant Dis. 99:1727-1731.

Recent fungicide efficacy studies indicated that brown rot fruit rot at harvest, caused by Monilinia fructicola, was being controlled by residual activity from protectant fungicides applied during the time between bloom and the preharvest fruit ripening period. To determine the extent of this residue, a simple in vivo bioassay was developed by assaying $M$. fructicola spore germination directly on sampled fruit. A $1.5-\mathrm{cm}$ section of clear flexible tubing was placed upright on harvested fruit to create a small incubation well. After the tubing-fruit interface was sealed using silicon grease, a suspension of $M$. fructicola conidia was pipetted into the well. The spores were suspended in a buffer-substrate medium consisting of $0.025 \mathrm{M}$ potassium phosphate, $0.1 \%$ sucrose, and $0.1 \%$ yeast extract. A rubber stopper

with an aeration hole was inserted into the well's top and the fruit was placed in an incubator at $25^{\circ} \mathrm{C}$. Results of a time-course study indicated that the optimal conidial incubation time was $6 \mathrm{~h}$. Bioassay sensitivity was evaluated by examining test results from varying concentrations of captan fungicide. Results indicated that captan residue levels as low as one-thousandth the standard field rate could be detected using spore germination as the predictor. Fitting of the logistic decline model to the data created a standard curve to allow quantitative estimation of fungicide residue based on observed level of spore germination. A modified version of the bioassay, which can be used to detect carbohydrate or nutrient sources on the fruit surface, was also demonstrated.
\end{abstract}

In commercial peach (Prunus persica L. Batch) orchards located in the eastern United States, the management of diseases with fungicides can be divided into four periods of control. During the dormant period, one fungicide application is typically made in late fall or late winter prior to bud-swell for management of leaf curl, caused by Taphrina deformans (Burk.) Tul. (Jones and Sutton 1996). Fungicide applications during the spring bloom period and the preharvest fruit ripening period are primarily made for management of brown rot blossom blight and fruit rot, respectively, caused by Monilinia fructicola (G. Winter) Honey (Anderson 1956; Ogawa et al. 1995). In total, two to three applications, typically using systemic or site-specific fungicides, are performed during each of these periods (Hamilton et al. 2013).

The fourth period for peach disease management, typically called the "cover spray" period, occurs between the bloom and preharvest periods. This period begins at initiation of shuck-split, the time when growth of the young fruit causes the surrounding calyx to split, exposing the fruit surface to infection for the first time. Fungicide applications during the cover spray period are made during this time for management of summer diseases such as peach scab (Hendrix 1995), rusty spot (Furman et al. 2003), and anthracnose (Hendrix 1995; Jones and Sutton 1996). Depending on the cultivar's harvest date, two to eight fungicide applications, generally at 10- to 14-day intervals, are performed during the cover spray period (Hamilton et al. 2013). However, unlike the bloom and preharvest applications, the cover sprays are typically protectant fungicides, most commonly captan or sulfur.

In a 2010 fungicide efficacy field study on 'Autumnglo' peach, one particular treatment program utilized captan for the bloom, shuck-split, and all eight cover sprays but lacked the final three preharvest applications (Lalancette, N. and McFarland, K. A. unpublished). Regardless of this latter omission, this program significantly reduced brown rot incidence by $60 \%$. In a similar 2012 treatment program on

Corresponding author: N. Lalancette; E-mail: lalancette @ njaes.rutgers.edu

*The $\boldsymbol{e}$-Xtra logo stands for "electronic extra" and indicates that three supplementary figures are published online.

Accepted for publication 29 April 2015.

http://dx.doi.org/10.1094/PDIS-02-15-0191-RE

(C) 2015 The American Phytopathological Society
'Suncrest' peach, the bloom sprays were followed by captan for the first three cover sprays and sulfur for the final four cover sprays (McFarland and Lalancette 2013). Although this program also lacked the preharvest applications, it significantly reduced brown rot incidence by $56 \%$. These results suggest that applications during the cover spray period provided control of brown rot at harvest. One possible explanation is that the early cover sprays were preventing the formation of quiescent infections on young fruit; however, this type of infection has not been reported or observed on peach in the eastern United States (Ogawa et al. 1995). Another explanation is that the bloom sprays reduced blossom blight canker incidence, thereby reducing inoculum for the fruit rot phase. However, the treatment trees in both studies were surrounded by nontreated buffer trees, so that inoculum was probably not limiting. Another, perhaps more plausible explanation was the build-up of fungicide residue on fruit from the repeated cover spray applications. The multitude of trichomes on peach fruit would increase the surface area and, therefore, the amount of fungicide retained, thereby providing continued protectant activity well into the preharvest fruit ripening period.

In order to corroborate the fungicide retention hypothesis, measurements of fungicide residue levels are needed during the preharvest period following application of cover sprays. If the hypothesis was true, these data would show that protectant fungicide residue levels were sufficient to inhibit spore germination. Published fungicide residue detection techniques include a variety of analytical methodologies involving gas chromatography, mass spectrometry, and solidphase extraction, among others (Blasco et al. 2002). However, most of these methods, which utilize equipment not typically found in pathology labs, require some type of extraction protocol and are primarily designed for detecting very low levels of pesticides in fruit and vegetables for food safety concerns. In contrast, our technique need only detect fungicide residue levels sufficient for spore mortality. Thus, the major objective of this study was to develop a simple method or bioassay that would use spore germination of the pathogen in question, $M$. fructicola, as the indicator of the residue level. In addition, to eliminate the need of residue extraction, the assay would be conducted directly on the fruit surface utilizing miniature "incubation wells". Secondary objectives of the study include determination of the bioassay's range of sensitivity, creation of standard curves, and examination of the fruit effect on spore germination (absent of fungicide residue). Initial results of this study have been previously reported (Lalancette et al. 2014). 


\section{Materials and Methods}

Fruit samples. The study was conducted during the 2013 growing season. Fruit used in developing and testing the bioassay were obtained from 4-year-old and 17-year-old experimental peach orchards located at the Rutgers Agricultural Research and Extension Center, Bridgeton, NJ. Trees in the younger block consisted of 'Bounty' peach grafted on 'Baily' rootstock, while those in the older orchard consisted of Autumnglo grafted on 'Lovell' rootstock. No fungicide sprays were applied to the trees throughout the season. Insecticide applications were applied as needed according to commercial recommendations (Hamilton et al. 2013).

Bioassay technique. Harvested mature Bounty fruit were held stationary in the laboratory by placing them sideways on beakers or jars having openings slightly smaller than the diameter of the fruit. A 1.5-cm section of clear, flexible tubing (Tygon R-3603 Laboratory Tubing; Saint-Gobain Performance Plastics Corp., Akron, $\mathrm{OH}$ ) with an inside diameter of $12.7 \mathrm{~mm}$ and wall thickness of $1.6 \mathrm{~mm}$ was then placed upright on the fruit side (cheek) to create a miniature incubation well. Once on the fruit, the tubing was gently given a $90^{\circ}$ turn to aid in creating a seal. Because this region of the fruit possessed some curvature, and because fruit trichomes prevented a flush union, the tubing-fruit interface was sealed using silicon vacuum grease (High Vacuum Grease; Dow Corning Corp., Midland, MI). This compound, chosen because of its inert properties, was applied to the tubing edge and outer periphery of the joint. When applied properly, all grease should be external to the well, with none visible on the inside fruit surface. Using this approach, one well was placed on each fruit.

Test inoculum consisted of conidia harvested from 7-day-old cultures of a single-spore isolate of $M$. fructicola (Mf012) grown on potato dextrose agar (PDA) at 20 to $25^{\circ} \mathrm{C}$. Relative to other isolates, Mf012 has average growth and sporulates well in culture. The conidia were collected by spraying the fungal colonies with cold $\left(5^{\circ} \mathrm{C}\right)$ deionized water using a DeVilbiss atomizer set at $52 \mathrm{kPa}$ (DeVilbiss Health Care, Inc., Somerset, PA). The spore suspension was then centrifuged (speed 5 for $2 \mathrm{~min}$, IEC Clinical Centrifuge 801; Damon/IEC Division, Needham Hts, MA) to concentrate the conidia and remove any contaminating stimulant. After a second centrifugation wash with cold deionized water, the spores were resuspended in a cold $\left(5^{\circ} \mathrm{C}\right)$ buffer-substrate medium consisting of $0.025 \mathrm{M}$ potassium phosphate plus $0.1 \%$ sucrose and $0.1 \%$ yeast extract (pH 6.8). Finally, the concentration of the spore suspension was adjusted to 50,000 conidia/ml using spore counts made with a hemacytometer. If not used immediately, the suspension was placed in the refrigerator at $5^{\circ} \mathrm{C}$ or on ice to prevent premature germination of the spores. Past studies showed the germination process to be very slow at $5^{\circ} \mathrm{C}$ (Weaver 1950); nevertheless, suspensions were not held more than $1 \mathrm{~h}$ at this temperature.

To initiate the bioassay, $1.0 \mathrm{ml}$ of the cold conidial suspension was pipetted into each incubation well on each fruit. Because cuticle of the fruit epidermis and trichomes tends to be hydrophobic, the suspension was mechanically agitated by dispensing and retrieving it several times with the pipette to insure good contact with the fruit surface. This action also mixes any fungicide residue on the fruit surface with the inoculum. Following agitation, the wells were immediately capped by gently inserting a rubber stopper (number 00) into the well opening. The stoppers were shortened to prevent them from touching the suspension and a 2-mm-diameter hole was drilled for aeration and pressure equalization. After examination for leaks, the fruit and attached wells were placed in an unlit incubator at $25^{\circ} \mathrm{C}$ for incubation. Because the volume of the cold inoculum in each well was relatively small $(1.0 \mathrm{ml})$, it was assumed that it reached $25^{\circ} \mathrm{C}$ quickly, especially because the fruit and well were initially at room temperature (20 to $22^{\circ} \mathrm{C}$ ).

After a 6-h incubation period (determined below), fruit were removed from the incubator and assessed for conidia germination using a compound microscope at $\times 200$ magnification. Before taking a sample, the spore suspensions in the wells were gently mixed with the pipette by alternately withdrawing and dispensing the medium. In total, 100 conidia were examined for each fruit, with percent germination as the dependent variable. A conidium was considered "germinated" when its germ tube was at least the length of the spore.

Time-course study. To determine the optimum incubation period, 25 nontreated Bounty fruit were harvested from the test orchard, set up using the above bioassay technique, and placed in an unlit incubator at $25^{\circ} \mathrm{C}$. Spore suspensions were then added to each fruit at 10-min intervals to allow time for germination assessment. At approximately 1.25 - to 1.5 -h intervals, five fruit were removed from the incubator and assessed for conidia germination using the bioassay technique. Thus, the experimental design consisted of five incubation durations with five replicate fruit per duration and a total of 500 conidia examined per duration.

For comparison purposes, in vitro positive and negative control treatments were executed along with the bioassay treatment. The positive control treatment consisted of incubation of the conidial suspension in flasks as opposed to the in vivo incubation for the bioassay. The negative control treatment was also incubated in flasks but the conidia were suspended in deionized water as opposed to the buffer-substrate medium used for both the bioassay and positive control. Inoculum for all three treatments was obtained from the same M. fructicola Mf012 colonies grown on PDA. The entire time-course study was repeated once for a total of two experimental replications.

Bioassay sensitivity. The ability of the bioassay to detect low levels of fungicide was examined in a separate study. Captan, a protectant fungicide commonly used by commercial growers, was chosen for this purpose. Mature peach fruit harvested from the nontreated Autumnglo trees were sprayed to run-off with six different concentrations of captan (Captan 80WDG; Arysta LifeScience, Cary, NC) ranging from $1 X$ to $10^{-4} X$, where $X=1.2 \mathrm{~g}$ a.i./liter. In field applications, the $1 X$ concentration, hereto referred to as "standard concentration", translates to $460 \mathrm{~g}$ of product per hectare ( $368 \mathrm{~g}$ a.i./ha) at a full dilute volume of 307 liters/ha ( $2.5 \mathrm{lb}$. of product per 200 gal per acre).

Ten replicate fruit were treated for each captan concentration. Fruit were allowed to dry for $24 \mathrm{~h}$ prior to setup and execution of the bioassay. Based on results of the time-course study, an incubation period of $6 \mathrm{~h}$ was utilized. In total, 100 conidia from each incubation well were inspected for germination, resulting in examination of 1,000 conidia per captan concentration. The dependent variable was calculated as $R G=G_{T R T} / G_{N T C}$, where $R G=$ relative spore germination, $\mathrm{G}_{\mathrm{TRT}}=$ percent germination of the treatment, and $\mathrm{G}_{\mathrm{NTC}}=$ percent germination of the nontreated control.

Standard curve. The fungicide concentrations used in the sensitivity study, which were arranged as a base 10 logarithmic series, spanned the entire range of inhibition by captan. Thus, the same data could be used to generate a standard curve for predicting fungicide concentration (C) as a function of RG. Because the graph of linear RG plotted against $\log _{10}(\mathrm{C})$ depicts a reverse or declining sigmoid curve, the relationship was modeled using the logistic decline function $\mathrm{RG}=a+\left[b /\left(1+e^{[c(\mathrm{LC}-d)]}\right)\right]$, where $\mathrm{LC}=\log _{10}(\mathrm{C})$ and parameters $a, b, c$, and $d$ represent the model's minimum, maximum $-a$, shape, and inflection point on the abscissa, respectively. The model was fit to the data using the gauss-newton iterative method in the nonlinear regression procedure (PROC NLIN) of the Statistical Analysis System (v9.3; SAS Institute, Cary NC). Observed and standardized residuals were examined for goodness of fit.

Fruit effects. A small study was conducted to examine the possible stimulatory contribution from fruit to germination of conidia. Four mature, ripe fruit were harvested from a nonsprayed Autumnglo tree. As described in the bioassay technique, incubation wells were set up on two of the fruit with the standard buffer-substrate as the medium for the M. fructicola inoculum. Incubation wells were also set up on the remaining two fruit but the inoculum medium consisted of plain, deionized water. A third negative control treatment consisted of in vitro incubation of the $M$. fructicola inoculum in deionized water in Erlenmeyer flasks. As described for the bioassay technique, all treatments were incubated for $6 \mathrm{~h}$ in an unlit incubator at $25^{\circ} \mathrm{C}$ and percent conidia germination was determined by examining 100 to 125 spores per replicate. Data were subjected to an analysis of variance 
and treatment mean comparison using the Waller-Duncan $K$-ratio $t$ test.

\section{Results}

Time course study. Graphical plots of the results for the bioassay treatment revealed the classic sigmoidal growth pattern expected for the process of conidial germination (Fig. 1). The rate of germination was initially slow during the first $2.7 \mathrm{~h}$, resulting in only 7 to $16 \%$ conidia germination. However, a rapid increase was observed between 2.7 and $5.7 \mathrm{~h}$ of incubation, eventually producing 79 to $90 \%$ germination. No further increase in germination was observed between 5.7 and $7.2 \mathrm{~h}$ for the bioassay replicates. Given these findings, an incubation period of $6 \mathrm{~h}$ was deemed adequate for the bioassay technique.

The rate of germination was somewhat slower initially for the positive control treatment, particularly replicate 1 , relative to the bioassay results (Fig. 1). Furthermore, the percent germination for this control continued to increase during the last $2 \mathrm{~h}$ of incubation, eventually reaching 95 to $98 \%$. However, these differences are not considered significant, and the overall results from the positive control treatment, an in vitro assay utilizing the same buffer-substrate medium, mostly mimicked the results observed for the bioassay. In contrast, when conidia were not provided for the buffer-substrate medium, as with the negative control treatment, little or no germination was observed after $7.2 \mathrm{~h}$ of incubation (Fig. 1). These results confirm the importance of nutrition or $\mathrm{pH}$ for germination of M. fructicola conidia.

Bioassay sensitivity. A plot of relative conidia germination against the log of fungicide concentration produced the expected declining sigmoid pattern for both experimental replicates (Fig. 2). Except for results at the $0.01 X$ fungicide concentration, the data points for each replicate were nearly identical, with overlap of all standard error bars. At the highest $(1 X)$ and lowest $\left(10^{-4} X\right)$ fungicide concentrations, germination relative to the control ranged from 0 to 0.001 and 0.94 to 0.95 , respectively, thereby indicating that the full range of bioassay sensitivity was examined in the study.

Based on results of the sensitivity study, the bioassay has the ability to detect captan concentrations on fruit at one-hundredth to onethousandth of the standard concentration (Fig. 2). This detection range translates into 1.2 to $12 \mathrm{mg}$ a.i./liter. The bioassay was most sensitive between the $0.1 X$ and $0.01 X$ concentrations, the region having the greatest rate of change. In contrast, the bioassay has no sensitivity or

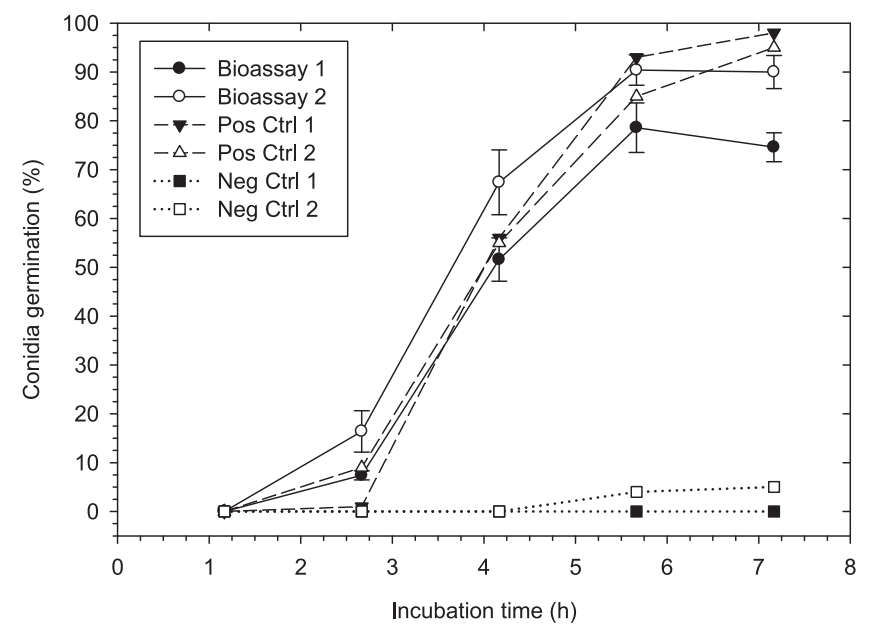

Fig. 1. Progression of conidia germination of Monilinia fructicola as a function of incubation time at constant $25^{\circ} \mathrm{C}$ for the bioassay and control treatments in the time-course study. The positive control consisted of in vitro germination in flasks with the buffer-substrate medium, while the negative control consisted of in vitro germination in flasks with deionized water as the medium. Each data point is based on observations of 500 conidia (five incubation wells or flasks $\times 100$ conidia); vertical bars are standard errors of the means. Two replications of each timecourse were performed for each treatment. lack of predictability (zero slope) at high $(1 X$ to $0.1 X)$ or very low $\left(10^{-3} X\right.$ to $\left.10^{-4} X\right)$ fungicide concentrations.

Standard curve. When fitting the logistic decline function to the data via nonlinear regression, the overall model effect was highly significant (Table 1). The standard errors of the model coefficients were relatively low for the $b, c$, and $d$ parameters, indicating a good fit to the data. The coefficients of variation (standard error/estimate $\times 100$ ) for these model parameters were $4.3,14.6$, and $2.6 \%$, respectively. In contrast, the $a$ parameter estimate had a very high standard error relative to its value. However, this parameter represents the minimum or lower asymptote of the model, which theoretically approaches zero, and the confidence interval does indeed contain zero. Based on the coefficient of determination, calculated as $1-$ SSE/SST, the model described $99 \%$ of the variation in the data.

Residual analysis showed a very good fit to the data, as was also evident from a fit of the predicted to the observed data (Fig. 3). Furthermore, the $95 \%$ confidence interval for predicted observations was most narrow in the range of greatest bioassay sensitivity ( $\mathrm{LC}=-1.0$ to -2.0 ), thereby indicating a high degree of precision when used as a standard curve in this region. Much less predictive precision was possible at high or very low fungicide concentrations; however, these regions are of less concern.

Fruit effects. Results of the analysis of variance showed that there was a significant treatment effect $(F=140.7, P=0.0011)$. Furthermore, variation within treatments was minimal, as indicated by similarity between the replicate results (Table 2). Comparison of the overall means revealed significant differences among all three treatments. The intermediate level of germination for conidia incubated in water on the fruit surface indicated that the fruit provided a stimulatory effect, although one that was limited relative to the positive control.

\section{Discussion}

A bioassay technique was successfully developed which utilizes in vivo germination of $M$. fructicola conidia to determine the biologically active concentration of fungicide residue on peach fruit surfaces. The placement of incubation wells directly on the fruit epidermis and use of in situ spore germination obviated the need for any residue extraction and its associated error from less-thanperfect fungicide recovery. The predicted range of residue levels, which are derived from germination-concentration standard curves, are directly relevant to plant disease control considerations. That

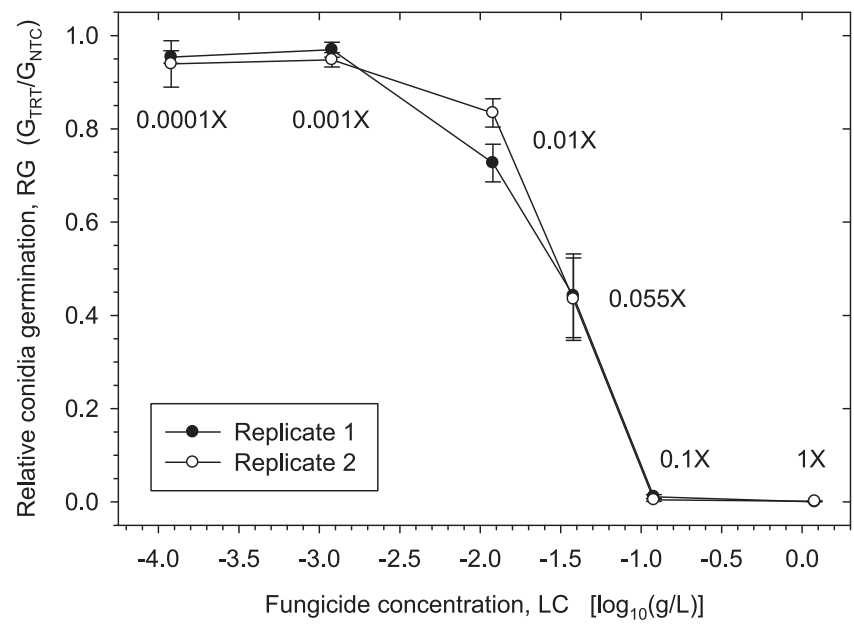

Fig. 2. Sensitivity of Monilinia fructicola conidia germination using the bioassay to varying concentrations of captan residue deposited on mature peach fruit. The dependent variable, relative germination, was calculated as percent germination on the treated fruit $\left(\mathrm{G}_{T R T}\right)$ /percent germination on nontreated fruit $\left(\mathrm{G}_{\mathrm{NTC}}\right)$. Each data point of each experimental replicate was based on observations of 1,000 conidia (10 fruit $\times 100$ conidia/fruit); vertical bars are standard errors of the means. Absolute levels of fungicide concentration are depicted on the graph as proportions of the $1 X$ rate (captan at $1.2 \mathrm{~g} /$ /iter); fungicide was applied to run-off on peach fruit. 
is, fungicide concentrations within the active range of the standard curve, where the slope is not equal to zero, indicate partial protectant activity and the need for action. Those predicted residue concentrations above or below the active range are either more than adequate or wholly insufficient for disease control, respectively. Within the context of plant disease control, there is no need to detect very low levels of residue, as would be required when assaying fruit for human consumption. These features of the bioassay certainly allow for practical applications, such as timing fungicide sprays based on residue levels. However, the duration of the technique (approximately 1 day) and need to assay many fruit would probably prohibit any large scale testing for multiple commercial enterprises. In its present form, the bioassay seems most appropriate as a research tool.

The bioassay's sensitivity is a function of (i) the pathogen used as an indicator, (ii) the incubation temperature, (iii) the inside diameter of the incubation wells, (iv) the volume of inoculum added to the wells, and (v) the concentration of conidial inoculum. Conidia of some pathogens may germinate over a broader concentration range of some fungicides, or require more or less fungicide to achieve a given level of germination inhibition. Variation within the pathogen genotype can be addressed by using a single-spore isolate having average growth characteristics. The incubation temperature used for the bioassay, $25^{\circ} \mathrm{C}$, was near optimum for M. fructicola (Weaver 1950). Less-than-optimum temperatures could possibly be compensated for by increasing the incubation period. However, suboptimum temperatures could also reduce the range of germination response, thereby limiting the bioassay's sensitivity. Clearly, use of wells with larger diameters or smaller inoculum volumes would increase the fungicide concentration in the inoculum suspension, thereby allowing detection of lower residue levels. Higher conidial densities can result in lower doses per spore, thereby altering the germination curve (McCallan et al. 1941). Consequently, the recommended concentration for in vitro spore germination studies was 50,000 spores $/ \mathrm{ml}$, which was adopted for the bioassay (American Phytopathological Society, Committee on Standardization of Fungicidal Tests 1943). However, minimal differences in germination were observed in a separate captan study comparing bioassay inoculum concentrations of 100,000 and 500,000 conidia $/ \mathrm{ml}$ to the 50,000 conidia/ml standard. Regardless of how these factors influence the bioassay's sensitivity, standardization of technique using the described or similar protocol will allow relative comparisons of different fungicides as well as temporal comparison of residues from the same fungicide.

If the disease in question is incited by the pathogen used as an indicator in the bioassay (e.g., brown rot caused by M. fructicola), then the percent germination data can be utilized directly in the study. Unless there is some other reason for predicting fungicide residue levels, there would be no need to generate and employ standard curves for each of the fungicides in the study. As with M. fructicola, many plant-pathogenic fungi are relatively easy to grow in vitro and readily sporulate in culture, making them good candidates for use as indicators in the bioassay. However, some pathogens (e.g. Fusicladium carpophilum) take much longer to culture in vitro, making them less amenable as indicators. Under these circumstances, standard germination-fungicide concentration curves would need to be generated once for the difficult-to-culture pathogen. In all future experiments, an easy-to-culture indicator pathogen could then be used in the bioassay to predict fungicide levels to be input into the pathogen's standard curve. One caveat to this approach is that the sensitivity range of the indicator fungus and the pathogen will need to mostly overlap; otherwise, some translations will not be possible.

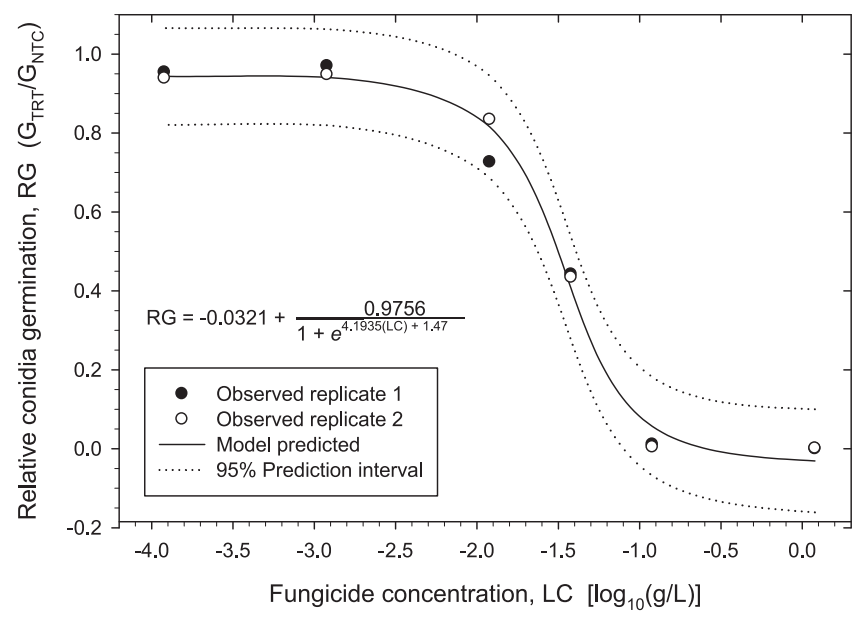

Fig. 3. Standard curve quantifying relative conidia germination of Monilinia fructicola as a function of captan concentration using the bioassay technique. Logistic decline model parameters were derived from nonlinear regression analysis on pooled data from both experimental replicates. The dependent variable was calculated as percent germination on the treated fruit $\left(\mathrm{G}_{T R T}\right) /$ percent germination on nontreated fruit $\left(\mathrm{G}_{\mathrm{NTC}}\right)$. Each data point of each experimental replicate was based on observations of 1,000 conidia (10 fruit $\times 100$ conidia/fruit). Captan fungicide was applied to run-off on each fruit sample.

Table 2. Stimulatory effect of mature, ripe fruit on germination of Monilinia fructicola conidia relative to positive in vivo control and negative in vitro control

\begin{tabular}{lcccc}
\hline \multicolumn{2}{c}{ Incubation treatment } & & \multicolumn{2}{c}{ Conidia germination $(\%)$} \\
\cline { 5 - 5 } Location & Medium & & Replicate & Mean $^{\mathbf{z}}$ \\
\hline Fruit/well & Buffer-substrate & & 92.2 & $93.3 \mathrm{a}$ \\
& & & 94.4 & \\
Fruit/well & Deionized water & & 62.9 & $69.9 \mathrm{~b}$ \\
& & & 76.8 & \\
Flask & Deionized water & & 0.0 & $0.5 \mathrm{c}$ \\
& & 0.9 & \\
\hline
\end{tabular}

${ }^{\mathrm{z}}$ Treatment means followed by the same letter are not significantly differen according to the Waller-Duncan $K$-ratio $t$ test $(K$-ratio $=100, P \leq 0.05)$.

Table 1. Nonlinear regression analysis results from modeling relative conidia germination as a function of $\log _{10}$ of captan fungicide concentration (LC)

\begin{tabular}{|c|c|c|c|c|c|c|c|c|}
\hline \multicolumn{3}{|c|}{ Parameter $^{y}$} & \multicolumn{2}{|c|}{$95 \% \mathrm{CI}^{\mathrm{z}}$} & \multirow[b]{2}{*}{ df } & \multirow[b]{2}{*}{ Mean square } & \multirow[b]{2}{*}{$F$ value } & \multirow[b]{2}{*}{$P>F$} \\
\hline Name & Estimate & SE & Lower & Upper & & & & \\
\hline$a$ & -0.0321 & 0.0315 & -0.1048 & 0.0405 & $\ldots$ & $\ldots$ & $\ldots$ & $\ldots$ \\
\hline$b$ & 0.9756 & 0.0416 & 0.8796 & 1.0716 & $\ldots$ & $\ldots$ & $\ldots$ & $\ldots$ \\
\hline$c$ & 4.1935 & 0.6124 & 2.7813 & 5.6057 & $\ldots$ & $\ldots$ & $\ldots$ & $\ldots$ \\
\hline$d$ & -1.47 & 0.0383 & -1.5583 & -1.3818 & $\ldots$ & $\ldots$ & $\ldots$ & $\ldots$ \\
\hline \multicolumn{9}{|l|}{ ANOVA } \\
\hline Model & $\ldots$ & $\ldots$ & $\ldots$ & $\ldots$ & 3 & 0.65 & 286.96 & $<0.0001$ \\
\hline Error & $\ldots$ & $\ldots$ & $\ldots$ & & 8 & 0.00227 & $\ldots$ & $\ldots$ \\
\hline
\end{tabular}


Nevertheless, this modification of the bioassay would allow one to easily collect residue data and determine its efficacy for difficultto-culture pathogens.

The time-course study was necessary to determine the optimum length of the incubation period. Germination was first observed for some spores after 2 to $3 \mathrm{~h}$, and this timing agrees with past observations (Byrd and Willetts 1977). However, allowing time for the majority of conidia to germinate improves the sensitivity of the bioassay, because the entire range from 0 to near $100 \%$ germination can be utilized. Based on the logistic growth response, this optimum in germination was attained when the sigmoidal growth curve reached its upper asymptote at about $6 \mathrm{~h}$. Although longer incubation periods would theoretically result in similar germination rates, there was concern that conidia with longer germ tubes may be more likely to adhere to the epidermis and trichomes, thereby altering the percentage of germinated spores sampled from the incubation well. Because $M$. fructicola is primarily a wound pathogen and infection through direct penetration is not common (Byrd and Willetts 1977), loss of germinated spores through infection was not considered to be significant. Nevertheless, once the 6-h limit is reached, all fungal growth can be halted by either placing the peach samples under cold conditions $\left(5^{\circ} \mathrm{C}\right)$ or by adding $10 \%$ formalin at $0.5 \%$ (vol/vol; $5 \mu \mathrm{l} /$ well).

M. fructicola conidia do not have sufficient carbohydrate reserves to germinate in the absence of exogenous nutrients (Naqvi and Good 1957). When conidia were transferred to distilled water via a brush (to avoid nutrient transfer from the medium), less than $10 \%$ germinated (Peterson 1941); this phenomenon was observed with our negative control in the time-course study. Consequently, after harvesting and washing the spores in a centrifugation step, the conidia in our bioassay are incubated in a buffer-substrate medium that includes sucrose and yeast extract as carbohydrate and protein sources, respectively. This approach allows the inoculum to be standardized across multiple executions of the bioassay, and insures that only the fungicide residue is limiting germination, not the lack of nutrients. However, it is also clear that the requirement for exogenous nutrients by M. fructicola can be exploited in a modified version of the bioassay that simply employs water as the incubation medium. The fruit effect study demonstrated that the modified bioassay could be used to detect the presence of exogenous nutrients, presumably carbohydrates leaching from the fruit as it matures and ripens (Fourie and Holz 1998). This activity by the fruit may relate directly to ontogenetic fruit susceptibility (Populer 1978).

Because the bioassay employs spore germination as its indicator of fungicide residue, only protectant fungicide activity can be investigated. This is not a major limitation for examining fungicides employed during the peach cover spray period, because these materials are typically protectants. Indeed, initial studies examining residues from these cover sprays have shown good correlation between bioassay results and brown rot incidence at harvest (unpublished data). The bioassay can still be useful for those site-specific fungicides that often move systemically in plant tissues but nevertheless have protectant activity. Some portion of the applied fungicide often remains on the fruit surface, where its activity can be assayed. Actually, in a time-course study on slow moving fungicides, it may be possible to track the declining surface residues and, therefore, declining protectant activity as the fungicide gradually moves into the plant tissue.
The bioassay technique should be applicable to a wide variety of plant pathosystems on many fruit and vegetable crops. The size of the incubation wells could be selected to be smaller or larger to accommodate different fruit sizes and geometries. However, very small wells may be too difficult to handle, especially when installing the rubber stopper as a cap. Also, if the fruit has too much curvature, the fruit-well interface may be too difficult to seal with the silicone grease, although it may be possible to cut the well tubing to match the profile of the fruit surface. In this case, all wells need to be cut with the same profile in order for the incubation area to remain constant. Finally, the indicator pathogen needs to readily produce conidia in vitro, not produce appressoria that would affix it to the suscept, and have relatively short germination times so that multiple treatments can easily be assayed using the same batch of inoculum.

\section{Acknowledgments}

This research was funded in part by the New Jersey Agricultural Experiment Station. We thank L. Blaus for her technical assistance in the later stages of the study.

\section{Literature Cited}

American Phytopathological Society, Committee on Standardization of Fungicidal Tests. 1943. The slide-germination method of evaluating protectant fungicides. Phytopathology 33:627-632.

Anderson, H. W. 1956. Pages 189-205 in: Diseases of Fruit Crops. McGraw-Hill Book Co., New York.

Blasco, C., Pico, Y., Manes, J., and Font, G. 2002. Determination of fungicide residues in fruits and vegetables by liquid chromatography - atmospheric pressure chemical ionization mass spectrometry. J. Chromatogr. A 947:227-235.

Byrd, R. J. W., and Willetts, H. J. 1977. Pages 46, 87-94 in: The Brown Rot Fungi of Fruit: Their Biology and Control. Pergamon Press, New York.

Fourie, J. F., and Holz, G. 1998. Effects of fruit and pollen exudates on growth of Botrytis cinerea and infection of plum and nectarine fruit. Plant Dis. 82:165-170.

Furman, L. A., Lalancette, N., and White, J. F., Jr. 2003. Peach rusty spot epidemics: Management with fungicide, effect on fruit growth, and the incidence -lesion density relationship. Plant Dis. 87:1477-1486.

Hamilton, G. C., Heckman, J. R., Lalancette, N., Majek, B. A., Nielsen, A., and Ward, D. L. 2013. Peach and nectarine spray guide. Pages 62-89 in: The New Jersey Commercial Tree Fruit Production Guide. N. J. Agric. Exp. Stn. Rutgers Coop. Ext. Bull. E002.

Hendrix, F. F., Jr. 1995. Scab. Pages 11-12 in: Compendium of Stone Fruit Diseases. J. M. Ogawa, E. I. Zehr, G. W. Bird, D. F. Ritchie, K. Uriu, and J. K. Uyemoto, eds. American Phytopathological Society. St. Paul, MN.

Jones, A. L. and Sutton, T. B. 1996. Diseases of tree fruits in the east. Mich. State Univ. Ext. NCR Ext. Bull. 45:63, 76.

Lalancette, N., Gager, J., and McFarland, K. A. 2014. A simple in vivo bioassay for estimating fungicide residues on peach fruit. (Abstr.) Phytopathology 104:S1.3

McCallan, S. E. A., Wellman, R. H., and Wilcoxon, F. 1941. An analysis of factors causing variation in spore germination tests of fungicides. III. Slope of toxicity curves, replicate tests, and fungi. Contrib. Boyce Thompson Inst. 12:49-78.

McFarland, K. A. and Lalancette, N. 2013. Management of peach scab with Quadris Top and Inspire Super, 2012. Online publication. Plant Dis. Manage. Rep. 7:STF019.

Naqvi, S. H. Z., and Good, H. M. 1957. Studies of the aging of conidia of Monilinia fructicola (Wint.) Honey: I. Germination rates and longevity. Can. J. Bot. 35:635-645

Ogawa, J. M., Zehr, E. I., and Biggs, A. R. 1995. Brown Rot. Pages 7-10 in Compendium of Stone Fruit Diseases. J. M. Ogawa, E. I. Zehr, G. W. Bird, D. F. Ritchie, K. Uriu, and J. K. Uyemoto, eds. American Phytopathological Society, St. Paul, MN.

Peterson, P. D. 1941. The spore-germination method of evaluating fungicides. Phytopathology 31:1108-1116.

Populer, C. 1978. Changes in host susceptibility with time. Pages 239-262 in: Plant Disease, An Advanced Treatise, Vol. II, How Disease Develops in Populations. J. G. Horsfall and E. B. Cowling, eds. Academic Press, New York.

Weaver, L. O. 1950. Effect of temperature and relative humidity on occurrence of blossom blight of stone fruits. Phytopathology 40:1136-1153. 